\title{
The Evolution of Harmonic Progression Analysis: Ultimate CAT
}

\author{
Authors: Carmine Cataldo \\ Submitted: $\quad 24$. September 2018 \\ Published: $\quad 29$. September 2018 \\ Volume: $\quad 5$ \\ Issue: 7 \\ Affiliation: Independent Researcher, $\mathrm{PhD}$ in Mechanical Engineering, MD in \\ Disciplines of Jazz and Improvisation (Jazz Piano), Battipaglia \\ (SA), Italy \\ Languages: English \\ Keywords: $\quad$ CAT, Cataldo Advanced Transformations, Chord Progression \\ Analysis, Harmonic Substitutions, Jazz \\ DOI: $\quad 10.17160 /$ josha.5.7.469
}

\section{Abstract:}

In this article we introduce an improved version of CAT (Cataldo Advanced Transformations). The purpose of CAT fundamentally lies in providing musicians with a reliable instrument that may effectively assist them in carrying out chord progressions analysis. The correct application of CAT allows to convert any harmonic progression, net of some very rare exceptions, into a mere sequence of plagal and perfect cadences. Amongst other features, the improved version of CAT, unlike the previous one, takes also into account Modal Interchange and Tonicization.

\section{JOSHA Jumnalosimene, Humanities and Arts}




\title{
The Evolution of Harmonic Progression Analysis: Ultimate CAT
}

\section{Carmine Cataldo}

$\mathrm{PhD}$ in Mechanical Engineering, MD in Disciplines of Jazz and Improvisation (Jazz Piano), Battipaglia (SA), Italy

\begin{abstract}
In this article we introduce an improved version of CAT (Cataldo Advanced Transformations). The purpose of CAT fundamentally lies in providing musicians with a reliable instrument that may effectively assist them in carrying out chord progressions analysis. The correct application of $C A T$ allows to convert any harmonic progression, net of some very rare exceptions, into a mere sequence of plagal and perfect cadences. Amongst other features, the improved version of $C A T$, unlike the previous one, takes also into account Modal Interchange and Tonicization.
\end{abstract}

\section{Keywords}

CAT, Cataldo Advanced Transformations, Chord Progression Analysis, Harmonic Substitutions, Jazz.

\section{Introduction}

Net of a single exception (a routine named "Structure Reduction"), the whole method is based on the mere application of a series of (inverse) harmonic transformations [1] [2]. The above-mentioned transformations, herein introduced in a considerably improved version, turn out to be nothing but inverse chord substitutions characterized by specific conditions and restrictions. As elsewhere underlined [7] [8], the method arises from the analysis of a considerable number of chord progressions, devoting particular (although not exclusive) attention to traditional jazz compositions. A significant improvement of $C A T$ has been achieved by conducting an extremely thorough analysis of a huge amount of LEGO Bricks (public domain harmonic patterns) [5] [6]. Unlike the previous version [3] [4], the one herein introduced has no limitation concerning the key (any song written in both major and minor key can be analysed), exploits a more rigorous definition of "Similitude", and takes into consideration "Modal Interchange" (comparing 35 scales/modes) and "Tonicization" (actually not to be regarded as a real substitution, but rather as a "Harmonic Enrichment") [7] [8]. The time signature must always be imagined as being equal to 4/4. For example, even if we deal with a $3 / 4$, we have to consider four pulses per measure (four beats per bar): each beat, in this case, will be characterized by a duration equivalent to a dotted quaver.

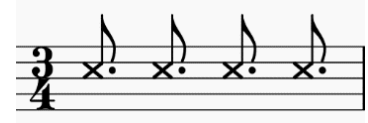

\section{KEY Selection and Writing of the SCALE and HARMONIZATION VECTORS}

If we denote with $X$ a generic note belonging to the Chromatic scale, and with $t$ a whole tone interval, we can represent the Ionian and the Aeolian scales as vectors [9] [10], denoted by $s^{I o n}$ and $s^{A e o}$ respectively [11] [12]. Similarly, we can consider two vectors, denoted by $h^{I o n}$ and $h^{A e o}$, whose components are the seventh chords that arise from the harmonization of the above-mentioned scales.

(X) MAJOR Key

$$
\boldsymbol{s}^{\operatorname{Ion}}(X)=\left[\begin{array}{c}
X \\
X+t \\
X+2 t \\
X+\frac{5}{2} t \\
X+\frac{7}{2} t \\
X+\frac{9}{2} t \\
X+\frac{11}{2} t
\end{array}\right]
$$

(X) MINOR Key

$$
\boldsymbol{h}^{A e o}(X)=\left[\begin{array}{c}
X m 7 \\
\langle X+t\rangle m 7 b 5 \\
\left\langle X+\frac{3}{2} t\right\rangle \text { maj7 } \\
\left\langle X+\frac{5}{2} t\right\rangle m 7 \\
\left\langle X+\frac{7}{2} t\right\rangle m 7 \\
\langle X+4 t\rangle m a j 7 \\
\langle X+5 t\rangle 7
\end{array}\right]
$$




\section{STRUCTURE REDUCTION}

Structure Reduction - Without "Structure Reduction" a correct application of CAT (Cataldo Advanced Transformations) is de facto impossible. Very simply, the number of bars and the duration of the chords are iteratively halved. The procedure is stopped the moment in which even a single chord characterized by a duration equal to a beat appears. "Structure Reduction" is applied every time it is possible, so as to obtain the highest simplification level. [3] [4]

Very concisely, we have:

$$
\begin{gathered}
n_{\max }=\text { current number of chords } \\
k_{\max }=\text { current number of bars } \\
a_{n}=n-\text { th chord } \\
T\left(a_{n}\right)=\text { current duration of the } n-\text { th chord } \\
\forall n \in\left(1, n_{\max }\right) \quad T\left(a_{n}\right)>1 \text { beat and } \frac{k_{\max }}{2} \in N \Longrightarrow T\left(a_{n}\right) \stackrel{\text { red. }}{\longrightarrow} \frac{T\left(a_{n}\right)}{2} \text { and } k_{\max } \stackrel{\text { red. }}{\longrightarrow} \frac{k_{\max }}{2}
\end{gathered}
$$

\begin{tabular}{|c|c|c|c|c|c|c|c|}
\hline$X=C$ & $\mathrm{~h}^{1}$ & $h^{2}$ & $h^{3}$ & $\mathrm{~h}^{4}$ & $h^{5}$ & $\mathrm{~h}^{6}$ & $h^{7}$ \\
\hline Ionian & Cmaj7 & $\mathrm{Dm} 7$ & $\mathrm{Em} 7$ & Fmaj7 & G7 & $\mathrm{Am} 7$ & $\mathrm{Bm} 7 \mathrm{~b} 5$ \\
\hline Dorian & $\mathrm{Cm} 7$ & Dm7 & $E^{b}$ maj7 & F7 & $\mathrm{Gm} 7$ & Am7b5 & $\mathrm{B}^{\mathrm{b}} \mathrm{maj} 7$ \\
\hline Phrygian & $\mathrm{Cm} 7$ & $\mathrm{D}^{\mathrm{b}} \mathrm{maj} 7$ & $\mathrm{E}^{\mathrm{b} 7}$ & Fm7 & Gm7b5 & $\mathrm{A}^{\mathrm{b}}$ maj7 & $\mathrm{B}^{\mathrm{b}} \mathrm{m} 7$ \\
\hline Lydian & Cmaj7 & D7 & $\mathrm{Em} 7$ & $\mathrm{~F}^{\#} \mathrm{~m} 7 \mathrm{~b} 5$ & Gmaj7 & $\mathrm{Am} 7$ & $\mathrm{Bm} 7$ \\
\hline Mixolydian & $\mathrm{C} 7$ & Dm7 & Em7b5 & Fmaj7 & $\mathrm{Gm} 7$ & Am7 & $\mathrm{B}^{\mathrm{b}} \mathrm{maj} 7$ \\
\hline Aeolian & $\mathrm{Cm} 7$ & $\operatorname{Dm} 7 \mathrm{~b} 5$ & $E^{b}$ maj7 & Fm7 & $\mathrm{Gm} 7$ & $\mathrm{~A}^{\mathrm{b}}$ maj7 & $\mathrm{B}^{\mathrm{b} 7}$ \\
\hline Locrian & $\mathrm{Cm} 7 \mathrm{~b} 5$ & $\mathrm{D}^{\mathrm{b}}$ maj7 & $\mathrm{E}^{\mathrm{b}} \mathrm{m} 7$ & $\mathrm{Fm} 7$ & $\mathrm{G}^{\mathrm{b}} \mathrm{maj} 7$ & $\mathrm{~A}^{\mathrm{b} 7}$ & $\mathrm{~B}^{\mathrm{b}} \mathrm{m} 7$ \\
\hline Ipoionian & $\mathrm{Cm}^{\Delta}$ & Dm7 & $E^{b}$ maj $7 \# 5$ & F7 & G7 & Am7b5 & $\mathrm{Bm} 7 \mathrm{~b} 5$ \\
\hline Dorian b2 & $\mathrm{Cm} 7$ & $\mathrm{D}^{\mathrm{b}}$ maj7\#5 & $\mathrm{E}^{\mathrm{b} 7}$ & F7 & $\mathrm{Gm} 7 \mathrm{~b} 5$ & Am7b5 & $\mathrm{B}^{\mathrm{b}} \mathrm{m}^{\Delta}$ \\
\hline Lydian Augmented & Cmaj7\#5 & D7 & E7 & $\mathrm{F} \# \mathrm{~m} 7 \mathrm{~b} 5$ & $\mathrm{G}^{*} \mathrm{~m} 7 \mathrm{~b} 5$ & $\mathrm{Am}^{\Delta}$ & $\mathrm{Bm} 7$ \\
\hline Lydian Dominant & $\mathrm{C} 7$ & D7 & $\mathrm{Em} 7 \mathrm{~b} 5$ & $\mathrm{~F}^{\#} \mathrm{~m} 7 \mathrm{~b} 5$ & $\mathrm{Gm}^{\Delta}$ & $\mathrm{Am} 7$ & $\mathrm{~B}^{\mathrm{b}} \mathrm{maj} 7 \# 5$ \\
\hline Mixolydian b6 & C7 & Dm7b5 & Em7b5 & $\mathrm{Fm}^{\Delta}$ & $\mathrm{Gm} 7$ & $\mathrm{~A}^{\mathrm{b}} \mathrm{maj} 7 \# 5$ & $\mathrm{~B}^{\mathrm{b} 7}$ \\
\hline Locrian \#2 & $\mathrm{Cm} 7 \mathrm{~b} 5$ & Dm7b5 & $E^{b} m^{\Delta}$ & Fm7 & $\mathrm{G}^{\mathrm{b}} \mathrm{maj} 7 \# 5$ & $\mathrm{~A}^{\mathrm{b} 7}$ & $\mathrm{~B}^{\mathrm{b} 7}$ \\
\hline Superlocrian & Cm7b5 & $\mathrm{D}^{b} \mathrm{~m}^{\Delta}$ & $\mathrm{E}^{\mathrm{b}} \mathrm{m} 7$ & $\mathrm{Fb}^{\mathrm{b}}$ maj7\#5 & $\mathrm{G}^{\mathrm{b}} 7$ & $\mathrm{~A}^{\mathrm{b} 7}$ & $\mathrm{~B}^{\mathrm{b}} \mathrm{m} 7 \mathrm{~b} 5$ \\
\hline Harmonic Minor & $\mathrm{Cm}^{\Delta}$ & $\operatorname{Dm} 7 \mathrm{~b} 5$ & $E^{b}$ maj7\#5 & Fm7 & G7 & $\mathrm{A}^{\mathrm{b}}$ maj7 & Bdim7 \\
\hline Locrian $\# 6$ & $\mathrm{Cm} 7 \mathrm{~b} 5$ & $\mathrm{D}^{\mathrm{b}}$ maj7 75 & $\mathrm{E}^{\mathrm{b}} \mathrm{m} 7$ & F7 & $\mathrm{G}^{\mathrm{b}}$ maj7 & $\operatorname{Adim} 7$ & $\mathrm{~B}^{\mathrm{b}} \mathrm{m}^{\Delta}$ \\
\hline Ionian Augmented & Cmaj7\#5 & $\operatorname{Dm} 7$ & E7 & Fmaj7 & $\mathrm{G}^{*} \operatorname{dim} 7$ & $\mathrm{Am}^{\Delta}$ & Bm7b5 \\
\hline Romanian & $\mathrm{Cm} 7$ & D7 & $E^{b}$ maj7 & $\mathrm{F}^{\#} \operatorname{dim} 7$ & $\mathrm{Gm}^{\Delta}$ & Am7b5 & $\mathrm{B}^{\mathrm{b}} \mathrm{maj} 7 \# 5$ \\
\hline Phrygian Dominant & $\mathrm{C} 7$ & $\mathrm{D}^{\mathrm{b}}$ maj 7 & $E \operatorname{dim} 7$ & $\mathrm{Fm}^{\Delta}$ & Gm7b5 & $\mathrm{A}^{\mathrm{b}} \mathrm{maj} 7 \# 5$ & $\mathrm{~B}^{\mathrm{b}} \mathrm{m} 7$ \\
\hline Lydian \#2 & Cmaj7 & $\mathrm{D}^{\prime} \operatorname{dim} 7$ & $\mathrm{Em}^{\Delta}$ & $\mathrm{F}^{ \pm} \mathrm{m} 7 \mathrm{~b} 5$ & Gmaj7\#5 & $\mathrm{Am} 7$ & B7 \\
\hline Ultralocrian & Cdim7 & $\mathrm{D}^{\mathrm{b}} \mathrm{m}^{\Delta}$ & $\mathrm{E}^{\mathrm{b}} \mathrm{m} 7 \mathrm{~b} 5$ & $\mathrm{~F}^{\mathrm{b}} \mathrm{maj} 7 \# 5$ & $\mathrm{G}^{\mathrm{b}} \mathrm{m} 7$ & $\mathrm{~A}^{\mathrm{b} 7}$ & $\mathrm{~B}^{\mathrm{bb}}$ maj 7 \\
\hline Harmonic Major & Cmaj7 & Dm7b5 & Em7 & Fm7 & G7 & $A^{b}$ maj7\#5 & Bdim7 \\
\hline Dorian b5 & $\mathrm{Cm} 7 \mathrm{~b} 5$ & $\operatorname{Dm} 7$ & $E^{b} \mathrm{~m} 7$ & F7 & $\mathrm{G}^{\mathrm{b}} \mathrm{maj} 7 \# 5$ & $\operatorname{Adim} 7$ & $\mathrm{~B}^{\mathrm{b}} \mathrm{maj} 7$ \\
\hline Superphrygian & $\mathrm{Cm} 7$ & $\mathrm{D}^{\mathrm{b}} \mathrm{m} 7$ & $E^{b} 7$ & $\mathrm{~F}^{\mathrm{b}} \mathrm{maj} 7 \# 5$ & $\mathrm{Gdim} 7$ & $\mathrm{~A}^{\mathrm{b}}$ maj7 & $\mathrm{B}^{\mathrm{b}} \mathrm{m} 7 \mathrm{~b} 5$ \\
\hline Lydian b3 & $\mathrm{Cm} 7$ & D7 & $E^{b}$ maj7\#5 & $\mathrm{F}^{\#} \operatorname{dim} 7$ & Gmaj7 & Am7b5 & $\mathrm{Bm} 7$ \\
\hline Mixolydian b2 & $\mathrm{C} 7$ & $\mathrm{D}^{\mathrm{b}} \mathrm{maj} 7 \# 5$ & $\operatorname{Edim} 7$ & Fmaj7 & $\mathrm{Gm} 7 \mathrm{~b} 5$ & $\mathrm{Am} 7$ & $\mathrm{~B}^{\mathrm{b}} \mathrm{m} 7$ \\
\hline Lydian Augmented \#2 & Cmaj7\#5 & $\mathrm{D}^{*} \operatorname{dim} 7$ & Emaj7 & $\mathrm{F} \# \mathrm{~m} 7 \mathrm{~b} 5$ & $\mathrm{G}^{\#} \mathrm{~m} 7$ & $\mathrm{Am} 7$ & B7 \\
\hline Locrian bb7 & Cdim7 & $\mathrm{D}^{\mathrm{b}}$ maj7 & $\mathrm{E}^{\mathrm{b}} \mathrm{m} 7 \mathrm{~b} 5$ & $\mathrm{Fm} 7$ & $\mathrm{G}^{\mathrm{b}} \mathrm{m} 7$ & $\mathrm{~A}^{\mathrm{b} 7}$ & $\mathrm{~B}^{\mathrm{bb}}$ maj $7 \# 5$ \\
\hline Double Harmonic & Cmaj7 & $\mathrm{D}^{\mathrm{b}} \mathrm{maj} 7$ & $\mathrm{Em}^{\operatorname{dim} 7}$ & $\mathrm{Fm}^{\Delta}$ & G7b5 & $\mathrm{A}^{\mathrm{b}} \mathrm{maj} 7 \# 5$ & $\mathrm{~B}(?)$ \\
\hline Lydian \#2 \#6 & Cmaj7 & $\mathrm{D}^{*} \mathrm{~m}^{\operatorname{dim} 7}$ & $\mathrm{Em}^{\Delta}$ & $\mathrm{F}^{\#} 7 \mathrm{~b} 5$ & Gmaj7\#5 & $\mathrm{A}^{\#}(?)$ & Bmaj7 \\
\hline Ultraphrygian & $\mathrm{Cm}^{\operatorname{dim} 7}$ & $\mathrm{D}^{\mathrm{b}} \mathrm{m}^{\Delta}$ & $\mathrm{E}^{\mathrm{b}} 7 \mathrm{~b} 5$ & $\mathrm{~F}^{\mathrm{b}} \mathrm{maj} 7 \# 5$ & $\mathrm{G}(?)$ & $\mathrm{A}^{\mathrm{b}}$ maj7 & $\mathrm{B}^{\mathrm{bb}}$ maj7 \\
\hline Hungarian Minor & $\mathrm{Cm}^{\Delta}$ & D7b5 & $E^{b}$ maj $7 \# 5$ & $\mathrm{~F}^{\#}(?)$ & Gmaj7 & $\mathrm{A}^{\mathrm{b}}$ maj7 & $\mathrm{Bm}^{\operatorname{dim} 7}$ \\
\hline Oriental & $\mathrm{C} 7 \mathrm{~b} 5$ & $\mathrm{D}^{\mathrm{b}} \mathrm{maj} 7 \# 5$ & $\mathrm{E}(?)$ & Fmaj7 & $\mathrm{G}^{\mathrm{b}} \mathrm{maj} 7$ & $\mathrm{Am}^{\operatorname{dim} 7}$ & $\mathrm{~B}^{\mathrm{b}} \mathrm{m}^{\Delta}$ \\
\hline Ionian Augmented \#2 & Cmaj7\#5 & $\mathrm{D}^{*}(?)$ & Emaj7 & Fmaj7 & $\mathrm{G}^{*} \mathrm{~m}^{\mathrm{dim} 7}$ & $\mathrm{Am}^{\Delta}$ & B7b5 \\
\hline Locrian bb3 bb7 & $C(?)$ & $\mathrm{D}^{\mathrm{b}} \mathrm{maj} 7$ & $\mathrm{E}^{\mathrm{bb}}$ maj7 & $\mathrm{Fm}^{\mathrm{dim} 7}$ & $\mathrm{G}^{\mathrm{b}} \mathrm{m}^{\Delta}$ & $\mathrm{A}^{\mathrm{b}} 7 \mathrm{~b} 5$ & $\mathrm{~B}^{\mathrm{bb}} \mathrm{maj} 7 \# 5$ \\
\hline
\end{tabular}

3. Inverse Substitutions (Modal Interchange and Similitude) involving AUGMENTED MAJOR SEVENTH CHORDS

Modal Interchange - Two chords that arise from the harmonization of two different scales characterized by the same tonic (generic parallel keys) are interchangeable if they are placed in the same position (if they represent the same harmonic degree). [7] [8] 
Similitude Substitution - Any Augmented Major Seventh Chord can be approximately identified with a Dominant Seventh Chord (provided with the flat thirteenth) distant an ascending major third. Any Minor Major Seventh Chord can be approximately identified with a Dominant Seventh Chord (provided with the sharp eleventh) distant an ascending perfect fourth [3] [4].

From now onwards, the examined chord (the one which must undergo substitution) will be denoted by $a_{n}$. The analysis must be carried out by starting from the final chord of the progression.

$$
\begin{aligned}
& \text { MAJOR Key } \\
& a_{n}=Y \operatorname{maj} 7 \# 5 \equiv Y^{\Delta} \# 5 \\
& s_{1}^{\text {Ion }} \text { maj7\#5 }=h_{1}^{\text {Lydian Augmented }} \stackrel{\text { mod. int. }}{\longleftarrow} h_{1}^{\text {Ion }} \\
& \left\langle s_{1}^{\text {Ion }}+\frac{t}{2}\right\rangle \text { maj7\#5 }=h_{2}^{\text {Dorian } b 2} \stackrel{\text { mod. int. }}{\longleftarrow} h_{2}^{\text {Ion }} \\
& s_{2}^{\text {Ion }} \text { maj7\#5 } \stackrel{\text { sim. }}{\longleftarrow}\left\langle s_{4}^{\text {Ion }}+\frac{t}{2}\right\rangle 7^{b 13} \cong\left\langle s_{4}^{\text {Ion }}+\frac{t}{2}\right\rangle 7 \\
& \left\langle s_{2}^{\text {Ion }}+\frac{t}{2}\right\rangle \text { maj7\#5 }=h_{3}^{\text {Ipo Ionian }} \stackrel{\text { mod. int. }}{\longleftarrow} h_{3}^{\text {Ion }}
\end{aligned}
$$

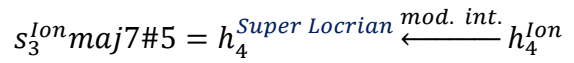

$$
\begin{aligned}
& s_{4}^{\text {Ion }} \operatorname{maj} 7 \# 5 \stackrel{\text { sim. }}{\longleftarrow} s_{6}^{\text {Ion }} 7^{b 13} \cong s_{6}^{\text {Ion }} 7
\end{aligned}
$$

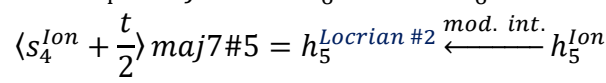

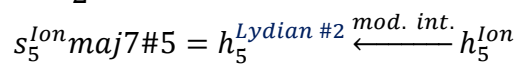

$$
\begin{aligned}
& \left\langle s_{5}^{\text {Ion }}+\frac{t}{2}\right\rangle \text { maj7\#5 }=h_{6}^{\text {Mixolydian } b 6} \underset{\text { mod. int. }}{\longleftarrow} h_{6}^{\text {Ion }}
\end{aligned}
$$

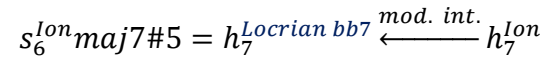

$$
\begin{aligned}
& \left\langle s_{6}^{\text {Ion }}+\frac{t}{2}\right\rangle \text { maj7\#5 }=h_{7}^{\text {Lydian Dominant }} \stackrel{\text { mod. int. }}{\longleftarrow} h_{7}^{\text {Ion }} \\
& s_{7}^{\text {Ion }} \text { maj7\#5 } \stackrel{\text { sim. }}{\longleftarrow}\left\langle s_{2}^{\text {Ion }}+\frac{t}{2}\right\rangle 7^{b 13} \cong\left\langle s_{2}^{\text {Ion }}+\frac{t}{2}\right\rangle 7
\end{aligned}
$$

$$
\begin{aligned}
& \text { MINOR Key } \\
& a_{n}=Y \operatorname{maj} 7 \# 5 \equiv Y^{\Delta} \# 5 \\
& s_{1}^{\text {Aeo }} \text { maj7\#5 }=h_{1}^{\text {Lydian Augmented }} \stackrel{\text { mod. int. }}{\longleftarrow} h_{1}^{\text {Aeo }}
\end{aligned}
$$

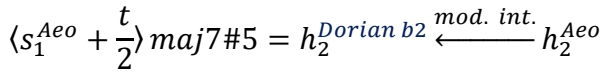

$$
\begin{aligned}
& s_{2}^{A e o} \text { maj7\#5 } \stackrel{\text { sim. }}{\longleftarrow}\left\langle s_{4}^{A e o}+\frac{t}{2}\right\rangle 7^{b 13} \cong\left\langle s_{4}^{A e o}+\frac{t}{2}\right\rangle 7
\end{aligned}
$$

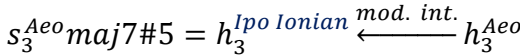

$$
\begin{aligned}
& \left\langle s_{3}^{\text {Aeo }}+\frac{t}{2}\right\rangle \text { maj7\#5 }=h_{4}^{\text {Super Locrian }} \stackrel{\text { mod. int. }}{\longleftarrow} h_{4}^{\text {Aeo }} \\
& s_{4}^{A e o} \operatorname{maj} 7 \# 5 \stackrel{\text { sim. }}{\longleftarrow}\left\langle s_{6}^{A e o}+\frac{t}{2}\right\rangle 7^{b 13} \cong\left\langle s_{6}^{A e o}+\frac{t}{2}\right\rangle 7
\end{aligned}
$$

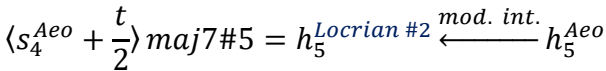

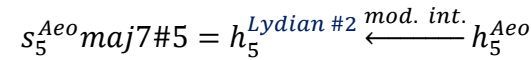

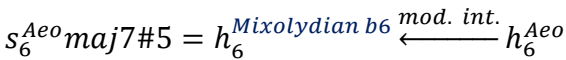

$$
\begin{aligned}
& \left\langle s_{6}^{A e o}+\frac{t}{2}\right\rangle \text { maj7\#5 }=h_{7}^{\text {Locrian } b b 7} \stackrel{\text { mod. int. }}{\longleftarrow} h_{7}^{\text {Aeo }} \\
& s_{7}^{\text {Ion }} \text { maj7\#5 }=h_{7}^{\text {Lydian Dominant }} \stackrel{\text { mod. int. }}{\longleftarrow} h_{7}^{\text {Aeo }} \\
& \left\langle s_{7}^{A e o}+\frac{t}{2}\right\rangle \operatorname{maj} 7 \# 5 \stackrel{\text { sim. }}{\longleftarrow} s_{3}^{A e o} 7^{b 13} \cong s_{3}^{A e o} 7
\end{aligned}
$$

4. Inverse Substitutions (Modal Interchange and Similitude)* involving MINOR MAJOR SEVENTH CHORDS

*See point 3 for the definitions of Modal Interchange and Similitude Substitution.

$$
\begin{aligned}
& \text { MAJOR Key } \\
& a_{n}=Y m^{\Delta} \\
& s_{1}^{\text {Ion }} m^{\Delta}=h_{1}^{\text {Ipo Ionian }} \stackrel{\text { mod. int. }}{\longleftarrow} h_{1}^{\text {Ion }}
\end{aligned}
$$

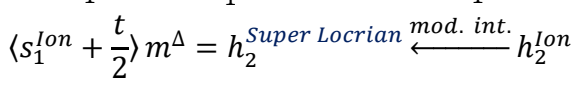

$$
\begin{aligned}
& s_{2}^{\text {Ion }} m^{\Delta} \stackrel{\text { sim. }}{\longleftarrow} h_{5}^{\text {Ion }} \stackrel{\# 11}{9} \cong h_{5}^{\text {Ion }}
\end{aligned}
$$

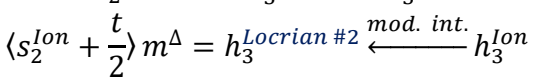

$$
\begin{aligned}
& s_{3}^{\text {Ion }} m^{\Delta}=h_{3}^{\text {Lydian } \# 2 ~} \stackrel{\text { mod. int. }}{\longleftarrow} h_{3}^{\text {Ion }} \\
& s_{4}^{\text {Ion }} m^{\Delta}=h_{4}^{\text {Mixolydian b6 }} \stackrel{\text { mod. int. }}{\longleftarrow} h_{4}^{\text {Ion }}
\end{aligned}
$$

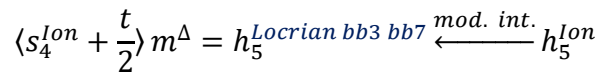

$$
\begin{aligned}
& s_{5}^{\text {Ion }} m^{\Delta}=h_{5}^{\text {Lydian Dominant }} \stackrel{\text { mod. int. }}{\longleftarrow} h_{5}^{\text {Ion }} \\
& \left\langle s_{5}^{\text {Ion }}+\frac{t}{2}\right\rangle m^{\Delta} \stackrel{\text { sim. }}{\longleftarrow}\left\langle s_{1}^{\text {Ion }}+\frac{t}{2}\right\rangle 7^{\# 11} \cong\left\langle s_{1}^{\text {Ion }}+\frac{t}{2}\right\rangle 7 \\
& s_{6}^{\text {Ion }} m^{\Delta}=h_{6}^{\text {Lydian Augmented }} \stackrel{\text { mod. int. }}{\longleftarrow} h_{6}^{\text {Ion }} \\
& \left\langle s_{6}^{\text {Ion }}+\frac{t}{2}\right\rangle m^{\Delta}=h_{7}^{\text {Dorian } b 2} \stackrel{\text { mod. int. }}{\longleftarrow} h_{7}^{\text {Ion }} \\
& s_{7}^{\text {Ion }} m^{\Delta} \stackrel{\text { sim. }}{\longleftarrow} s_{3}^{\text {Ion }}{ }_{7}^{\# 11} \cong s_{3}^{\text {Ion }} 7
\end{aligned}
$$

$$
\begin{aligned}
& \text { MINOR Key } \\
& a_{n}=Y m^{\Delta} \\
& s_{1}^{\text {Aeo }} m^{\Delta}=h_{1}^{\text {Ipo Ionian }} \stackrel{\text { mod. int. }}{\longleftarrow} h_{1}^{\text {Aeo }}
\end{aligned}
$$

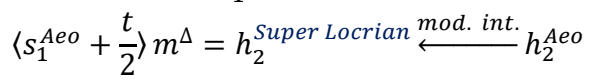

$$
\begin{aligned}
& s_{2}^{A e o} m^{\Delta} \stackrel{\text { sim. }}{\longleftarrow} s_{5}^{A e o} \stackrel{\# 11}{9} \cong s_{5}^{A e o} 7
\end{aligned}
$$

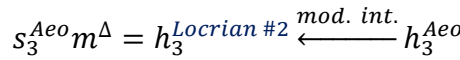

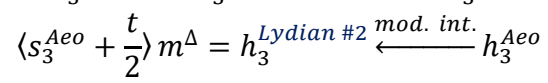

$$
\begin{aligned}
& s_{4}^{\text {Aeo }} m^{\Delta}=h_{4}^{\text {Mixolydian } b 6} \stackrel{\text { mod. int. }}{\longleftarrow} h_{4}^{\text {Aeo }}
\end{aligned}
$$

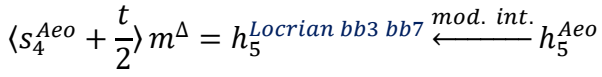

$$
\begin{aligned}
& s_{5}^{\text {Aeo }} m^{\Delta}=h_{5}^{\text {Lydian Dominant }} \stackrel{\text { mod. int. }}{\longleftarrow} h_{5}^{\text {Aeo }} \\
& s_{6}^{A e o} m^{\Delta} \stackrel{\text { sim. }}{\longleftarrow}\left\langle s_{1}^{A e o}+\frac{t}{2}\right\rangle 7^{\# 11} \cong\left\langle s_{1}^{A e o}+\frac{t}{2}\right\rangle 7 \\
& \left\langle s_{6}^{\text {Aeo }}+\frac{t}{2}\right\rangle m^{\Delta}=h_{6}^{\text {Lydian Augmented }} \stackrel{\text { mod. int. }}{\longleftarrow} h_{6}^{\text {Aeo }} \\
& s_{7}^{\text {Aeo }} m^{\Delta}=h_{7}^{\text {Dorian b2 }} \stackrel{\text { mod. int. }}{\longleftarrow} h_{7}^{\text {Aeo }} \\
& \left\langle s_{7}^{\text {Aeo }}+\frac{t}{2}\right\rangle m^{\Delta} \stackrel{\text { sim. }}{\longleftarrow}\left\langle s_{3}^{\text {Aeo }}+\frac{t}{2}\right\rangle 7^{\# 11} \cong\left\langle s_{3}^{\text {Aeo }}+\frac{t}{2}\right\rangle 7
\end{aligned}
$$


5. Inverse Substitutions (Modal Interchange* and Inverse Diminished Substitution) involving DIMINISHED CHORDS followed by Half-Diminished or Diminished Chords

Diminished Substitution - Any Dominant Seventh Chord, especially if provided with the flat ninth, can be replaced, even if it were to arise from a previous harmonic substitution, by a Diminished Chord distant a major third, a perfect fifth, a minor seventh or a flat ninth from the initial chord. All the above-mentioned intervals must be regarded as ascending [1] [2] [3] [4] [7] [8].

In other terms, if we denote with $P$ a generic note belonging to the Chromatic scale, we can write, with obvious meaning of the notation, as follows:

$$
\begin{gathered}
P 7^{(b 9)} \stackrel{\text { dim. }}{\longrightarrow} Y \operatorname{dim} 7 \\
Y=P+2 t+\frac{3 n}{2} t \quad n=0,1,2,3
\end{gathered}
$$

More explicitly, we have:

$$
P 7^{(b 9)} \stackrel{\operatorname{dim} .}{\longrightarrow}\left\{\begin{array}{l}
\langle P+2 t\rangle \operatorname{dim} 7 \quad n=0, \text { major third } \\
\left\langle P+\frac{7}{2} t\right\rangle \operatorname{dim} 7 \quad n=1, \text { perfect fifth } \\
\langle P+5 t\rangle \operatorname{dim} 7 \quad n=2, \text { minor seventh } \\
\left\langle P+\frac{13}{2} t\right\rangle \operatorname{dim} 7 \quad n=3, \text { flat ninth }
\end{array}\right.
$$

*See point 3 for the definition of Modal Interchange

$$
\begin{aligned}
& \text { MAJOR Key } \\
& \left\{a_{n}=Y \operatorname{dim} 7\right. \\
& \left\{\begin{array}{l}
a_{n+1}=Z m 7 b 5, Z \operatorname{dim} 7 \\
\text { a }
\end{array}\right. \\
& s_{1}^{\text {Ion }} \operatorname{dim} 7=h_{1}^{\text {Ultra Locrian }} \stackrel{\text { mod. int. }}{\longleftarrow} h_{1}^{\text {Ion }} \\
& \left\langle s_{1}^{\text {Ion }}+\frac{t}{2}\right\rangle \operatorname{dim} 7 \stackrel{\text { dim. }}{\longleftarrow} s_{6}^{\text {Ion }} 7^{b 9} \cong s_{6}^{\text {Ion }} 7 \\
& s_{2}^{\text {Ion }} \operatorname{dim} 7 \stackrel{\text { dim. }}{\longleftarrow}\left\langle s_{6}^{\text {Ion }}+\frac{t}{2}\right\rangle 7^{b 9} \cong\left\langle s_{6}^{\text {Ion }}+\frac{t}{2}\right\rangle 7
\end{aligned}
$$

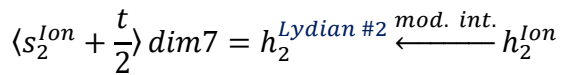

$$
\begin{aligned}
& s_{3}^{\text {Ion }} \operatorname{dim} 7=h_{3}^{\text {Phrygian Dominant }} \stackrel{\text { mod. int. }}{\longleftarrow} h_{3}^{\text {Ion }} \\
& s_{4}^{\text {Ion }} \operatorname{dim} 7 \stackrel{\text { dim. }}{\longleftarrow}\left\langle s_{1}^{\text {Ion }}+\frac{t}{2}\right\rangle 7^{\text {b9 }} \cong\left\langle s_{1}^{\text {Ion }}+\frac{t}{2}\right\rangle 7 \\
& \left\langle s_{4}^{\text {Ion }}+\frac{t}{2}\right\rangle \operatorname{dim} 7=h_{4}^{\text {Romanian }} \stackrel{\text { mod. int. }}{\longleftarrow} h_{4}^{\text {Ion }} \\
& s_{5}^{\text {Ion }} \operatorname{dim} 7=h_{5}^{\text {Super Phrygian }} \stackrel{\text { mod. int. }}{\longleftarrow} h_{5}^{\text {Ion }} \\
& \left\langle s_{5}^{\text {Ion }}+\frac{t}{2}\right\rangle \operatorname{dim} 7=h_{5}^{\text {Ionian Augmented }} \stackrel{\text { mod. int. }}{\longleftarrow} h_{5}^{\text {Ion }} \\
& s_{6}^{\text {Ion }} \operatorname{dim} 7=h_{6}^{\text {Locrian } \# 6} \underset{\text { mod. int. }}{\longleftarrow} h_{6}^{\text {Ion }} \\
& \left\langle s_{6}^{\text {Ion }}+\frac{t}{2}\right\rangle \operatorname{dim} 7 \stackrel{\text { dim. }}{\longleftarrow}\left\langle s_{4}^{\text {Ion }}+\frac{t}{2}\right\rangle 7^{b 9} \cong\left\langle s_{4}^{\text {Ion }}+\frac{t}{2}\right\rangle 7 \\
& s_{7}^{\text {Ion }} \operatorname{dim} 7=h_{6}^{\text {Harmonic Minor }} \stackrel{\text { mod. int. }}{\longleftarrow} h_{7}^{\text {Ion }}
\end{aligned}
$$

$$
\begin{aligned}
& \text { MINOR Key } \\
& a_{n}=Y \operatorname{dim} 7 \\
& \left\{\begin{array}{l}
a_{n+1}=Z m 7 b 5, Z \operatorname{dim} 7 \\
\text { dim }
\end{array}\right. \\
& s_{1}^{\text {Aeo }} \operatorname{dim} 7=h_{1}^{\text {Ultra Locrian }} \stackrel{\text { mod. int. }}{\longleftarrow} h_{1}^{\text {Aeo }} \\
& \left\langle s_{1}^{A e o}+\frac{t}{2}\right\rangle \operatorname{dim} 7 \stackrel{\text { dim. }}{\longleftarrow}\left\langle s_{6}^{A e o}+\frac{t}{2}\right\rangle 7^{b 9} \cong\left\langle s_{6}^{A e o}+\frac{t}{2}\right\rangle 7 \\
& s_{2}^{A e o} \operatorname{dim} 7 \stackrel{\operatorname{dim} .}{\longleftarrow} S_{7}^{A e o b 9} \cong s_{7}^{A e o} 7
\end{aligned}
$$

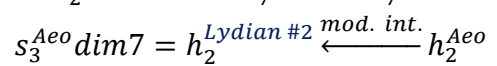

$$
\begin{aligned}
& \left\langle s_{3}^{\text {Aeo }}+\frac{t}{2}\right\rangle \operatorname{dim} 7=h_{3}^{\text {Phrygian Dominant }} \stackrel{\text { mod. int. }}{\longleftarrow} s_{3}^{\text {Aeo }} \\
& s_{4}^{A e o} \operatorname{dim} 7 \stackrel{\text { dim. }}{\longleftarrow}\left\langle s_{1}^{A e o}+\frac{t}{2}\right\rangle 7^{b 9} \cong\left\langle s_{1}^{A e o}+\frac{t}{2}\right\rangle 7 \\
& \left\langle s_{4}^{\text {Aeo }}+\frac{t}{2}\right\rangle \operatorname{dim} 7=h_{4}^{\text {Romanian }} \stackrel{\text { mod. int. }}{\longleftarrow} h_{4}^{\text {Aeo }} \\
& s_{5}^{\text {Aeo }} \operatorname{dim} 7=h_{5}^{\text {Super Phrygian }} \stackrel{\text { mod. int. }}{\longleftarrow} h_{5}^{\text {Aeo }} \\
& s_{6}^{\text {Aeo }} \operatorname{dim} 7=h_{5}^{\text {Ionian Augmented }} \stackrel{\text { mod. int. }}{\longleftarrow} h_{5}^{\text {Aeo }}
\end{aligned}
$$

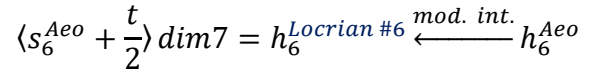

$$
\begin{aligned}
& s_{7}^{A e o} \operatorname{dim} 7 \stackrel{\text { dim. }}{\longleftarrow}\left\langle s_{4}^{A e o}+\frac{t}{2}\right\rangle 7^{b 9} \cong\left\langle s_{4}^{A e o}+\frac{t}{2}\right\rangle 7
\end{aligned}
$$

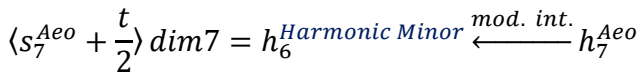

6. Inverse Diminished Substitutions involving DIMINISHED CHORDS not followed by Half-Diminished or Diminished Chords

\section{*See point 5 for the definition of Diminished Substitution}

Unlike the previous version of $C A T$ [3] [4], we herein consider two cases altogether: 
6.1. Inverse Diminished Substitutions involving Diminished chords followed by Dominant Seventh Chords

$$
\begin{aligned}
& a_{n}=Y \operatorname{dim} 7 \\
& \left\{\begin{array}{l}
a_{n+1}=Z 7 \\
a_{n} \in \operatorname{sub}^{\text {dim }}[Z 7]
\end{array} \Longrightarrow a_{n} \stackrel{\text { dim. }}{\longleftarrow} Z 7\right. \\
& \left\{\begin{array}{l}
a_{n+1}=Z 7 \\
a_{n} \in \operatorname{sub}^{d i m}\left[\left\langle Z+\frac{5}{2} t\right\rangle 7\right]
\end{array} \Longrightarrow a_{n} \stackrel{\text { dim. }}{\longleftarrow}\left\langle Z+\frac{5}{2} t\right\rangle 7\right. \\
& \left\{\begin{array}{l}
a_{n+1}=Z 7 \\
a_{n} \in \operatorname{sub}^{\text {dim }}\left[\left\langle Z+\frac{7}{2} t\right\rangle 7\right]
\end{array} \Longrightarrow a_{n} \stackrel{\text { dim. }}{\longleftarrow}\left\langle Z+\frac{7}{2} t\right\rangle 7\right.
\end{aligned}
$$

6.2. Inverse Diminished Substitutions involving Diminished chords followed by Major Seventh or Minor Seventh Chords

$$
\begin{aligned}
& a_{n}=Y \operatorname{dim} 7 \\
& \left\{\begin{array}{l}
a_{n+1}=Z m a j 7, Z m 7 \\
a_{n} \in \operatorname{sub}^{\text {dim }}\left[\left\langle Z+\frac{5}{2} t\right\rangle 7\right]
\end{array} \Longrightarrow a_{n} \stackrel{\text { dim. }}{\longleftarrow}\left\langle Z+\frac{5}{2} t\right\rangle 7\right. \\
& \left\{\begin{array}{l}
a_{n+1}=Z \operatorname{Zmaj} 7, Z m 7 \\
\left.a_{n} \in \operatorname{sub}^{\operatorname{dim}}\left[\left\langle Z+\frac{7}{2} t\right\rangle\right]\right]
\end{array} \Longrightarrow a_{n} \stackrel{\text { dim. }}{\longleftarrow}\left\langle Z+\frac{7}{2} t\right\rangle 7\right. \\
& \left\{\begin{array}{l}
a_{n+1}=Z \operatorname{Zmaj} 7, Z m 7 \\
a_{n} \in \operatorname{sub}^{\operatorname{dim}}\left[\left\langle Z+\frac{9}{2} t\right\rangle 7\right]
\end{array} \Longrightarrow a_{n} \stackrel{\text { dim. }}{\longleftarrow}\left\langle Z+\frac{9}{2} t\right\rangle 7\right.
\end{aligned}
$$

\section{CONTRACTION (Inverse EXPANSION)}

Expansion - Any Dominant Seventh Chord, by forgoing half of its duration, can be imagined as being preceded by a Minor Seventh or a Half-Diminished Chord distant a descending perfect fourth and characterized by a duration equal to half of the one of the initial chord. In other terms, any Dominant Seventh Chord can be converted into a half-cadence [3] [4].

$$
\left\{\begin{array}{l}
a_{n}=Y m 7, Y m 7 b 5 \\
a_{n+1}=\left\langle Y+\frac{5}{2} t\right\rangle 7 \\
a_{n}, a_{n+1} \in \operatorname{bar}_{k} \\
T\left(a_{n}\right)=T\left(a_{n+1}\right) \\
\text { beat }\left(a_{n}\right)=\text { on }
\end{array} \Longrightarrow a_{n}\left|a_{n+1} \stackrel{\text { exp. }}{\longleftarrow} a_{n+1}\right| a_{n+1}\right.
$$

\section{STRUCTURE REDUCTION*}

Note - Every time a Reduction has been carried out, it is necessary to evaluate the possibility of carry out Contractions the application of which, in turn, could make feasible another Structure Reduction, and so on. In other terms, we have a LOOP that can be stopped only if there are no conditions that make possible further Contractions and Structure Reductions [3] [4].

\section{*See point 2 for the definition of Structure Reduction}

9. Inverse Substitutions (Modal Interchange*) involving MAJOR SEVENTH CHORDS different from $h_{l}$ and $h_{4}$, if the Key is Major, or $h_{3}$ and $h_{6}$, if the Key is Minor (Major Seventh Chords that does not belong to the Harmonization Vector).

*See point 3 for the definition of Modal Interchange

$$
\begin{aligned}
& \text { MAJOR Key } \\
& a_{n}=Y \operatorname{maj} 7 \equiv Y^{\Delta} \neq h_{1}^{\text {Ion }}, h_{4}^{\text {Ion }} \\
& \left\langle s_{1}^{\text {Ion }}+\frac{t}{2}\right\rangle \text { maj7 }=h_{2}^{\text {Phrygian }} \stackrel{\text { mod. int. }}{\longleftarrow} h_{2}^{\text {Ion }}
\end{aligned}
$$

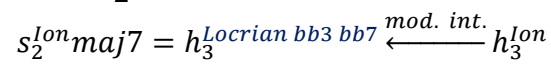

$$
\begin{aligned}
& \text { MINOR Key } \\
& a_{n}=Y \operatorname{maj} 7 \equiv Y^{\Delta} \neq h_{3}^{A e o}, h_{6}^{A e o} \\
& s_{1}^{\text {Aeo }} \text { maj7 }=h_{1}^{\text {Ionian }} \stackrel{\text { mod. int. }}{\longleftarrow} h_{1}^{\text {Aeo }} \\
& \left\langle s_{1}^{\text {Aeo }}+\frac{t}{2}\right\rangle \text { maj7 }=h_{2}^{\text {Phrygian }} \stackrel{\text { mod. int. }}{\longleftarrow} h_{2}^{\text {Aeo }}
\end{aligned}
$$




$$
\begin{aligned}
& \left\langle s_{2}^{\text {Ion }}+\frac{t}{2}\right\rangle \text { maj7 }=h_{3}^{\text {Dorian }} \stackrel{\text { mod. int. }}{\longleftarrow} h_{3}^{\text {Ion }}
\end{aligned}
$$

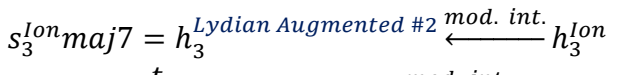

$$
\begin{aligned}
& \left\langle s_{4}^{\text {Ion }}+\frac{t}{2}\right\rangle \text { maj } 7=h_{5}^{\text {Locrian }} \stackrel{\text { mod. int. }}{\longleftarrow} h_{5}^{\text {Ion }} \\
& s_{5}^{\text {Ion }} \text { maj7 }=h_{5}^{\text {Lydian }} \stackrel{\text { mod. int. }}{\longleftarrow} h_{5}^{\text {Ion }} \\
& \left\langle s_{5}^{\text {Ion }}+\frac{t}{2}\right\rangle \text { maj7 }=h_{6}^{\text {Phrygian }} \stackrel{\text { mod. int. }}{\longleftarrow} h_{6}^{\text {Ion }} \\
& s_{6}^{\text {Ion }} \text { maj7 }=h_{7}^{\text {Ultra Locrian }} \stackrel{\text { mod. int. }}{\longleftarrow} h_{7}^{\text {Ion }} \\
& \left\langle s_{6}^{\text {Ion }}+\frac{t}{2}\right\rangle \text { maj7 }=h_{7}^{\text {Dorian }} \stackrel{\text { mod. int. }}{\longleftarrow} h_{7}^{\text {Ion }}
\end{aligned}
$$

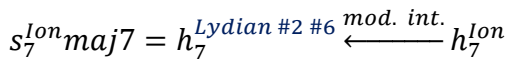

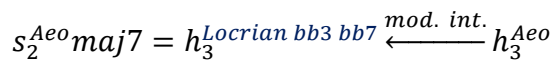

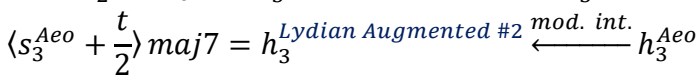

$$
\begin{aligned}
& s_{4}^{\text {Aeo }} \text { maj7 }=h_{4}^{\text {Ionian }} \stackrel{\text { mod. int. }}{\longleftarrow} h_{4}^{\text {Aeo }} \\
& \left\langle s_{4}^{\text {Aeo }}+\frac{t}{2}\right\rangle \text { maj7 }=h_{5}^{\text {Locrian }} \stackrel{\text { mod. int. }}{\longleftarrow} h_{5}^{\text {Aeo }} \\
& s_{5}^{\text {Aeo }} \text { maj7 }=h_{5}^{\text {Lydian }} \stackrel{\text { mod. int. }}{\longleftarrow} h_{5}^{\text {Aeo }} \\
& \left\langle s_{6}^{\text {Aeo }}+\frac{t}{2}\right\rangle \text { maj7 }=h_{7}^{\text {Ultra Locrian }} \stackrel{\text { mod. int. }}{\longleftarrow} h_{7}^{\text {Aeo }} \\
& s_{7}^{\text {Aeo }} \text { maj7 }=h_{7}^{\text {Dorian }} \stackrel{\text { mod. int. }}{\longleftarrow} h_{7}^{\text {Aeo }}
\end{aligned}
$$

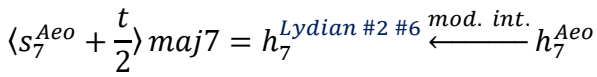

\section{CONTRACTIONS (Inverse EXPANSION) + STRUCTURE REDUCTION [LOOP]}

11. Inverse Substitutions (Modal Interchange and Similitude)* involving HALF - DIMINISHED CHORDS different from $h_{7}$, if the Key is Major, or $h_{2}$, if the Key is Minor (Half - Diminished Chords that does not belong to the Harmonization Vector).

\section{*See point 3 for the definitions of Modal Interchange and Similitude}

$$
\begin{aligned}
& \text { MAJOR Key } \\
& a_{n}=Y m 7 b 5 \equiv Y \emptyset \neq h_{7}^{\text {Ion }} \\
& \left\{\begin{array}{l}
a_{n}=\left\langle s_{4}^{\text {Ion }}+\frac{1}{2} t\right\rangle m 7 b 5 \\
a_{n+1}=s_{4}^{\text {Ion }} 7 \\
a_{n}, a_{n+1} \in \text { bar }_{k} \\
T\left(a_{n}\right)=T\left(a_{n+1}\right) \\
\text { beat }\left(a_{n}\right)=\text { on }
\end{array} \Longrightarrow a_{n}=a_{n}\right. \text { (no substitution) } \\
& \left\{\begin{array}{l}
a_{n}=s_{i}^{\text {Ion }} m 7 b 5 \\
a_{n+1}=\left\langle s_{i}^{\text {Ion }}+\frac{11}{2} t\right\rangle 7 \\
a_{n}, a_{n+1} \in \text { bar }_{k} \\
T\left(a_{n}\right)=T\left(a_{n+1}\right) \\
\operatorname{beat}\left(a_{n}\right)=\text { on }
\end{array} \Longrightarrow a_{n}=a_{n} \text { (no substitution) } i \neq 4,7\right. \\
& s_{1}^{\text {Ion }} m 7 b 5=h_{1}^{\text {Locrian }} \stackrel{\text { mod. int. }}{\longleftarrow} h_{1}^{\text {Ion }} \\
& \left\langle s_{1}^{\text {Ion }}+\frac{t}{2}\right\rangle m 7 b 5 \stackrel{\text { sim }}{\longleftarrow} s_{6}^{\text {Ion }} 7^{9} \cong s_{6}^{\text {Ion }} 7 \\
& s_{2}^{\text {Ion }} m 7 b 5=h_{2}^{\text {Aeolian }} \stackrel{\text { mod. int. }}{\longleftarrow} h_{2}^{\text {Ion }} \\
& \left\langle s_{2}^{\text {Ion }}+\frac{t}{2}\right\rangle m 7 b 5=h_{3}^{\text {Ultra Locrian }} \stackrel{\text { mod. int. }}{\longleftarrow} h_{3}^{\text {Ion }} \\
& s_{3}^{\text {Ion }} m 7 b 5=h_{3}^{\text {Mixolydian }} \stackrel{\text { mod. int. }}{\longleftarrow} h_{3}^{\text {Ion }} \\
& s_{4}^{\text {Ion }} m 7 b 5 \stackrel{\text { sim. }}{\longleftarrow}\left\langle s_{1}^{\text {Ion }}+\frac{t}{2}\right\rangle 7^{9} \cong\left\langle s_{1}^{\text {Ion }}+\frac{t}{2}\right\rangle 7 \\
& \left\langle s_{4}^{\text {Ion }}+\frac{t}{2}\right\rangle m 7 b 5=h_{4}^{\text {Lydian }} \stackrel{\text { mod. int. }}{\longleftarrow} h_{4}^{\text {Ion }} \\
& s_{5}^{\text {Ion }} m 7 b 5=h_{5}^{\text {Phrygian }} \stackrel{\text { mod. int. }}{\longleftarrow} h_{5}^{\text {Ion }} \\
& \left\langle s_{5}^{\text {Ion }}+\frac{t}{2}\right\rangle m 7 b 5=h_{5}^{\text {Lydian Augmented }} \stackrel{\text { mod. int. }}{\longleftarrow} h_{5}^{\text {Ion }} \\
& s_{6}^{\text {Ion }} m 7 b 5=h_{6}^{\text {Dorian }} \stackrel{\text { mod. int. }}{\longleftarrow} h_{6}^{\text {Ion }}
\end{aligned}
$$

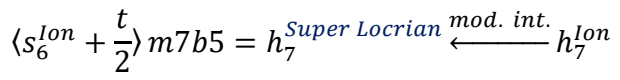

\section{MINOR Key}

$a_{n}=Y m 7 b 5 \equiv Y \varnothing \neq h_{2}^{A e o}$

$\left\{\begin{array}{l}a_{n}=\left\langle s_{6}^{A e o}+\frac{1}{2} t\right\rangle m 7 b 5 \\ a_{n+1}=s_{6}^{A e o} 7 \\ a_{n}, a_{n+1} \in \text { bar }_{k} \\ T\left(a_{n}\right)=T\left(a_{n+1}\right) \\ \text { beat }\left(a_{n}\right)=\text { on }\end{array} \Longrightarrow a_{n}=a_{n}\right.$ (no substitution)

$\left\{\begin{array}{l}a_{n}=s_{i}^{A e o} m 7 b 5 \\ a_{n+1}=\left\langle s_{i}^{A e o}+\frac{11}{2} t\right\rangle 7 \\ a_{n}, a_{n+1} \in \text { bar }_{k} \\ T\left(a_{n}\right)=T\left(a_{n+1}\right) \\ \text { beat }\left(a_{n}\right)=\text { on }\end{array} \Longrightarrow a_{n}=a_{n}\right.$ (no substitution) $i \neq 2,6$

$$
\begin{aligned}
& s_{1}^{\text {Aeo }} m 7 b 5=h_{1}^{\text {Locrian }} \stackrel{\text { mod. int. }}{\longleftarrow} h_{1}^{\text {Aeo }} \\
& \left\langle s_{1}^{A e o}+\frac{t}{2}\right\rangle m 7 b 5 \stackrel{\text { sim }}{\longleftarrow}\left\langle s_{6}^{A e o}+\frac{t}{2}\right\rangle 7^{9} \cong\left\langle s_{6}^{A e o}+\frac{t}{2}\right\rangle 7 \\
& s_{3}^{A e o} m 7 b 5=h_{3}^{\text {Ultra Locrian }} \stackrel{\text { mod. int. }}{\longleftarrow} h_{3}^{\text {Aeo }} \\
& \left\langle s_{3}^{\text {Aeo }}+\frac{t}{2}\right\rangle m 7 b 5=h_{3}^{\text {Mixolydian }} \stackrel{\text { mod. int. }}{\longleftarrow} h_{3}^{\text {Aeo }} \\
& s_{4}^{A e o} m 7 b 5 \stackrel{s i m}{\longleftarrow}\left\langle s_{1}^{A e o}+\frac{t}{2}\right\rangle 7^{9} \cong\left\langle s_{1}^{A e o}+\frac{t}{2}\right\rangle 7 \\
& \left\langle s_{4}^{\text {Aeo }}+\frac{t}{2}\right\rangle m 7 b 5=h_{4}^{\text {Lydian }} \stackrel{\text { mod. int. }}{\longleftarrow} h_{4}^{\text {Aeo }} \\
& s_{5}^{A e o} m 7 b 5=h_{5}^{\text {Phrygian }} \stackrel{\text { mod. int. }}{\longleftarrow} h_{5}^{\text {Aeo }} \\
& s_{6}^{\text {Aeo }} m 7 b 5=h_{5}^{\text {Lydian Augmented }} \stackrel{\text { mod. int. }}{\longleftarrow} h_{5}^{\text {Aeo }} \\
& \left\langle s_{6}^{\text {Aeo }}+\frac{t}{2}\right\rangle m 7 b 5=h_{6}^{\text {Dorian }} \stackrel{\text { mod. int. }}{\longleftarrow} h_{6}^{\text {Aeo }}
\end{aligned}
$$

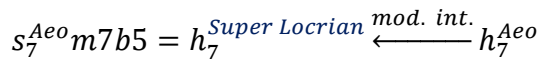

$$
\begin{aligned}
& \left\langle s_{7}^{\text {Aeo }}+\frac{t}{2}\right\rangle m 7 b 5=h_{7}^{\text {Ionian }} \stackrel{\text { mod. int. }}{\longleftarrow} h_{7}^{\text {Aeo }}
\end{aligned}
$$




\section{CONTRACTIONS (Inverse EXPANSION) + STRUCTURE REDUCTION [LOOP]}

13. Inverse Substitutions (Modal Interchange*) involving MINOR SEVENTH CHORDS different from $h_{2}, h_{3}$, and $h_{6}$, if the Key is Major, or $h_{1}, h_{4}$, and $h_{5}$, if the Key is Minor (Minor Seventh Chords that does not belong to the Harmonization Vector).

*See point 3 for the definition of Modal Interchange

$$
\begin{gathered}
\text { MAJOR Key } \\
a_{n}=Y m 7 \neq h_{2}^{I o n}, h_{3}^{I o n}, h_{6}^{\text {Ion }}
\end{gathered}
$$

$$
\left\{\begin{array}{l}
a_{n}=\left\langle s_{4}^{\text {Ion }}+\frac{1}{2} t\right\rangle m 7 \\
a_{n+1}=s_{4}^{\text {Ion }} 7 \\
a_{n}, a_{n+1} \in \text { bar }_{k} \\
T\left(a_{n}\right)=T\left(a_{n+1}\right) \\
\text { beat }\left(a_{n}\right)=\text { on }
\end{array} \Longrightarrow a_{n}=a_{n}\right. \text { (no substitution) }
$$$$
\left\{\begin{array}{l}
a_{n}=s_{i}^{\text {Ion }} m 7 \\
a_{n+1}=\left\langle s_{i}^{\text {Ion }}+\frac{11}{2} t\right\rangle 7 \\
a_{n}, a_{n+1} \in \text { bar }_{k} \\
T\left(a_{n}\right)=T\left(a_{n+1}\right) \\
\text { beat }\left(a_{n}\right)=\text { on }
\end{array} \Longrightarrow a_{n}=a_{n} \text { (no substitution) } i=1,5,7\right.
$$

$$
\begin{gathered}
s_{1}^{\text {Ion }} m 7=h_{1}^{\text {Aeolian }} \stackrel{\text { mod. int. }}{\longleftarrow} h_{1}^{\text {Ion }} \\
\left\langle s_{1}^{\text {Ion }}+\frac{t}{2}\right\rangle m 7=h_{2}^{\text {Super Phrygian }} \stackrel{\text { mod. int. }}{\longleftarrow} h_{2}^{\text {Ion }} \\
\left\langle s_{2}^{\text {Ion }}+\frac{t}{2}\right\rangle m 7=h_{3}^{\text {Locrian }} \stackrel{\text { mod. int. }}{\longleftarrow} h_{3}^{\text {Ion }} \\
s_{4}^{\text {Ion } m 7}=h_{4}^{\text {Aeolian }} \stackrel{\text { mod. int. }}{\longleftarrow} h_{4}^{\text {Ion }} \\
\left\langle s_{4}^{\text {Ion }}+\frac{t}{2}\right\rangle m 7=h_{5}^{\text {Ultra Locrian }} \stackrel{\text { mod. int. }}{\longleftarrow} h_{5}^{\text {Ion }} \\
s_{5}^{\text {Ion } m 7}=h_{5}^{\text {Aeolian }} \stackrel{\text { mod. int. }}{\longleftarrow} h_{5}^{\text {Ion }} \\
\left\langle s_{5}^{\text {Ion }}+\frac{t}{2}\right\rangle m 7=h_{5}^{\text {Lydian Augmented } \# 2 ~} \stackrel{\text { mod. int. }}{\longleftarrow} h_{5}^{\text {Ion }} \\
\left\langle s_{6}^{\text {Ion }}+\frac{t}{2}\right\rangle m 7=h_{7}^{\text {Phrygian }} \stackrel{\text { mod. int. }}{\longleftarrow} h_{7}^{\text {Ion }} \\
s_{7}^{\text {Ion }} m 7=h_{7}^{\text {Lydian }} \stackrel{\text { mod. int. }}{\longleftarrow} h_{7}^{\text {Ion }}
\end{gathered}
$$

MINOR Key

$$
a_{n}=Y m 7 \neq h_{1}^{A e o}, h_{4}^{A e o}, h_{5}^{A e o}
$$$$
\left\{\begin{array}{l}
a_{n}=\left\langle s_{6}^{A e o}+\frac{1}{2} t\right\rangle m 7 \\
a_{n+1}=s_{6}^{\text {Aeo }} 7 \\
a_{n}, a_{n+1} \in \text { bar }_{k} \\
T\left(a_{n}\right)=T\left(a_{n+1}\right) \\
\text { beat }\left(a_{n}\right)=\text { on }
\end{array} \Longrightarrow a_{n}=a_{n}\right. \text { (no substitution) }
$$$$
\left\{\begin{array}{l}
a_{n}=s_{i}^{A e o} m 7 \\
a_{n+1}=\left\langle s_{i}^{A e o}+\frac{11}{2} t\right\rangle 7 \\
a_{n}, a_{n+1} \in \text { bar }_{k} \\
T\left(a_{n}\right)=T\left(a_{n+1}\right) \\
\text { beat }\left(a_{n}\right)=\text { on }
\end{array} \Longrightarrow a_{n}=a_{n} \text { (no substitution) } i=2,3,7\right.
$$

$$
\begin{aligned}
& \left\langle s_{1}^{\text {Aeo }}+\frac{t}{2}\right\rangle m 7=h_{2}^{\text {Super Phrygian }} \stackrel{\text { mod. int. }}{\longleftarrow} h_{2}^{\text {Aeo }} \\
& s_{2}^{\text {Aeo }} \mathrm{m} 7=h_{2}^{\text {Ionian }} \stackrel{\text { mod. int. }}{\longleftarrow} h_{2}^{\text {Aeo }} \\
& s_{3}^{\text {Aeo }} \mathrm{m} 7=h_{3}^{\text {Locrian }} \stackrel{\text { mod. int. }}{\longleftarrow} h_{3}^{\text {Aeo }} \\
& \left\langle s_{3}^{\text {Aeo }}+\frac{t}{2}\right\rangle m 7=h_{3}^{\text {Ionian }} \stackrel{\text { mod. int. }}{\longleftarrow} h_{3}^{\text {Aeo }} \\
& \left\langle s_{4}^{\text {Aeo }}+\frac{t}{2}\right\rangle m 7=h_{5}^{\text {Ultra Locrian }} \stackrel{\text { mod. int. }}{\longleftarrow} h_{5}^{\text {Aeo }}
\end{aligned}
$$

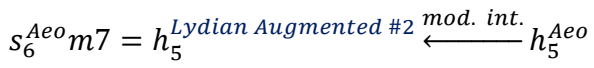

$$
\begin{aligned}
& \left\langle s_{6}^{\text {Aeo }}+\frac{t}{2}\right\rangle m 7=h_{2}^{\text {Ionian }} \stackrel{\text { mod. int. }}{\longleftarrow} h_{6}^{\text {Aeo }} \\
& s_{7}^{\text {Aeo }} \mathrm{m} 7=h_{7}^{\text {Phrygian }} \stackrel{\text { mod. int. }}{\longleftarrow} h_{7}^{\text {Aeo }} \\
& \left\langle s_{7}^{\text {Aeo }}+\frac{t}{2}\right\rangle m 7=h_{7}^{\text {Lydian }} \stackrel{\text { mod. int. }}{\longleftarrow} h_{7}^{\text {Aeo }}
\end{aligned}
$$

\section{CONTRACTIONS (Inverse EXPANSION) + STRUCTURE REDUCTION [LOOP]}

\section{5. (Inverse) TRITONE Substitutions}

Tritone Substitution - Any Dominant Seventh Chord, especially if altered, can be replaced, even if it were to arise from a previous harmonic substitution, by a chord of the same kind (a Dominant Seventh Chord) distant three whole tones from the initial chord.

Generally, if we denote with $P$ a generic note belonging to the Chromatic scale, and with $t$ a whole tone interval, we can write:

$$
\begin{aligned}
& X 7 \stackrel{\text { dim. }}{\longrightarrow} Y 7 \\
& Y=X+3 t \\
& a_{n}=Y 7 \\
& \left\{\begin{array}{l}
a_{n}=Y 7 \\
Y \neq s_{i}^{\text {Ion }}
\end{array} \Longrightarrow a_{n} \stackrel{\text { tri. }}{\longleftarrow}\langle Y+3 t\rangle 7 \quad i=1, \ldots, 7\right. \\
& \left\{\begin{array}{l}
a_{n}=Y 7 \\
Y \neq s_{i}^{\text {Aeo }}
\end{array} \Longrightarrow a_{n} \stackrel{\text { tri. }}{\leftarrow}\langle Y+3 t\rangle 7 \quad i=1, \ldots, 7\right.
\end{aligned}
$$




$$
\begin{gathered}
\left\{\begin{array}{l}
a_{n}=s_{4}^{\text {Ion }} 7 \\
a_{n-1}=h_{5}^{I o n},\left\langle s_{5}^{\text {Ion }}+3 t\right\rangle 7
\end{array} \Longrightarrow a_{n} \stackrel{\text { tri. }}{\longleftarrow} s_{7}^{\text {Ion }} 7\right. \\
\left\{\begin{array}{l}
a_{n}=s_{4}^{\text {Ion }} 7 \\
a_{n-1}=\left\langle s_{4}^{\text {Ion }}+\frac{1}{2} t\right\rangle m 7 b 5,\left\langle s_{4}^{\text {Ion }}+\frac{1}{2} t\right\rangle m 7
\end{array} \Longrightarrow a_{n} \stackrel{\text { tri. }}{\longleftarrow} s_{7}^{\text {Ion } 7}\right. \\
\left\{\begin{array}{l}
a_{n}=s_{7}^{\text {Ion }} 7 \\
a_{n-1}=s_{1}^{I o n} m 7 b 5, s_{1}^{I o n} m 7
\end{array} \Longrightarrow a_{n} \stackrel{\text { tri. }}{\longleftarrow} s_{4}^{\text {Ion }} 7\right.
\end{gathered}
$$

$$
\begin{gathered}
\left\{\begin{array}{l}
a_{n}=s_{2}^{A e o} 7 \\
a_{n-1}=h_{5}^{A e o}, s_{5}^{A e o} 7,\left\langle s_{5}^{A e o}+3 t\right\rangle 7
\end{array} \Longrightarrow a_{n} \stackrel{\text { tri. }}{\longleftarrow} s_{6}^{A e o} 7\right. \\
\left\{\begin{array}{l}
a_{n}=s_{2}^{A e o} 7 \\
a_{n-1}=s_{3}^{A e o} m 7 b 5, s_{3}^{A e o} m 7
\end{array} \Longrightarrow a_{n} \stackrel{\text { tri. }}{\longleftarrow} s_{6}^{A e o} 7\right. \\
\left\{\begin{array}{l}
a_{n}=s_{6}^{A e o} 7 \\
a_{n-1}=\left\langle s_{6}^{A e o}+\frac{1}{2} t\right\rangle m 7 b 5,\left\langle s_{6}^{I o n}+\frac{1}{2} t\right\rangle m 7
\end{array} \Longrightarrow a_{n} \stackrel{\text { tri. }}{\leftarrow} s_{2}^{A e o} 7\right.
\end{gathered}
$$

\section{CONTRACTIONS (Inverse EXPANSION) + STRUCTURE REDUCTION [LOOP]}

\section{SECONDARY DOMINANTS Inverse Substitutions}

Secondary Dominant Substitution - Any chord, even if it were to arise from a previous harmonic substitution, can be converted into a Dominant Seventh Chord [1] [2] [3] [4] [7] [8].

$$
\begin{gathered}
\text { MAJOR Key } \\
a_{n}=Y 7 \\
a_{n}=s_{i}^{\text {Ion }} 7 \Longrightarrow a_{n} \stackrel{\text { sec. dom. }}{\longleftarrow} h_{i}^{\text {Ion }} \quad i \neq 5
\end{gathered}
$$

$$
\begin{gathered}
\text { MINOR Key } \\
a_{n}=Y 7 \\
a_{n}=s_{i}^{\text {Aeo }} 7 \Longrightarrow a_{n} \stackrel{\text { sec. dom. }}{\longleftarrow} h_{i}^{\text {Aeo }} \quad i \neq 5,7
\end{gathered}
$$

The Secondary Dominants Inverse Substitutions involving $s_{5}$ are procrastinated in order to facilitate possible Contractions.

\section{CONTRACTIONS (Inverse EXPANSION) + STRUCTURE REDUCTION [LOOP]}

19. (Inverse) DIATONIC Substitutions

Diatonic Substitution - Two chords that arise from the harmonization of the same scale are interchangeable if the distance between them (between the roots) is equal to a diatonic third (both ascending and descending) [1] [2] [3] [4] [7] [8].

\section{Substitutions Chart - MAJOR Key}

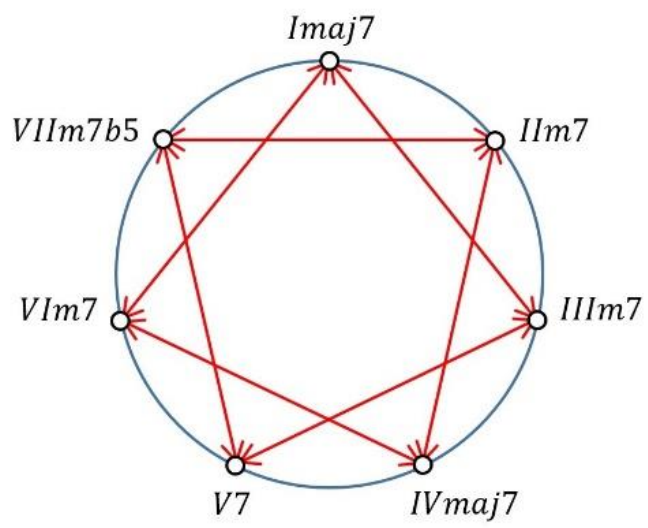

\section{Substitutions Chart - MINOR Key}

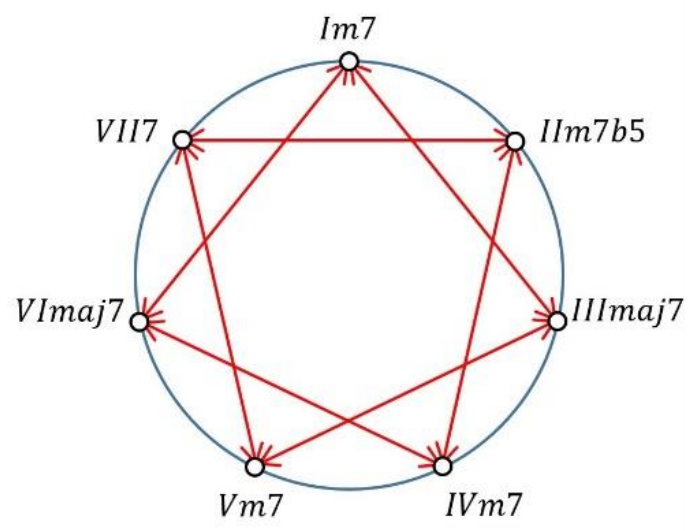

19.1. (Inverse) DIATONIC Substitutions involving $h_{6}$

$$
\begin{gathered}
\text { MAJOR Key } \\
a_{n}=h_{6}^{\text {Ion }} \Longrightarrow a_{n} \stackrel{\text { dia. }}{\longleftarrow} h_{1}^{\text {Ion }}
\end{gathered}
$$


19.2. (Inverse) DIATONIC Substitutions involving $h_{3}$

$$
\begin{aligned}
& \text { MAJOR Key } \\
& \left\{\begin{array}{l}
a_{n}=h_{3}^{\text {Ion }} \\
a_{n+1}=a_{n-1}=h_{1}^{\text {Ion }}
\end{array} \Longrightarrow a_{n} \stackrel{\text { dia. }}{\longleftarrow} h_{1}^{\text {Ion }}\right. \\
& \left\{\begin{array}{l}
a_{n}=h_{3}^{\text {Ion }} \\
a_{n+1} \neq h_{2}^{\text {Ion }}, h_{4}^{\text {Ion }} \Longrightarrow a_{n} \stackrel{\text { dia. }}{\longleftarrow} h_{5}^{\text {Ion }} \\
\text { beat }\left(a_{n}\right)=\text { off }
\end{array}\right. \\
& \left\{\begin{array}{l}
a_{n}=h_{3}^{\text {Ion }} \\
a_{n+1}=h_{2}^{\text {Ion }}, h_{4}^{\text {Ion }} \\
a_{n+2}=h_{5}^{\text {Ion }} \\
a_{n+1}, a_{n+2} \in \text { bar } \\
\text { beat }\left(a_{n}\right)=\text { off }
\end{array} \Longrightarrow a_{n} \stackrel{\text { dia. }}{\longleftarrow} h_{5}^{\text {Ion }}\right. \\
& \left\{\begin{array}{l}
a_{n}=h_{3}^{\text {Ion }} \\
a_{n+1} \neq h_{2}^{\text {Ion }}, h_{4}^{\text {Ion }} \\
a_{n-1}=h_{2}^{\text {Ion }}, h_{4}^{\text {Ion }}, h_{7}^{\text {Ion }} \\
a_{n-1}, a_{n} \in \text { bar }_{k}
\end{array} \Longrightarrow a_{n} \stackrel{\text { dia. }}{\longleftarrow} h_{5}^{\text {Ion }}\right. \\
& \text { otherwise: } a_{n}=h_{3}^{\text {Ion }} \stackrel{\text { dia. }}{\longleftarrow} h_{1}^{\text {Ion }}
\end{aligned}
$$

$$
\begin{aligned}
& \text { MINOR Key } \\
& \left\{\begin{array}{l}
a_{n}=h_{3}^{\text {Aeo }} \\
a_{n+1}=a_{n-1}=h_{1}^{\text {Aeo }}
\end{array} \Longrightarrow a_{n} \stackrel{\text { dia. }}{\longleftarrow} h_{1}^{\text {Aeo }}\right. \\
& \left\{\begin{array}{l}
a_{n}=h_{3}^{A e o} \\
a_{n+1} \neq h_{2}^{A e o}, h_{4}^{A e o} \Longrightarrow a_{n} \stackrel{\text { dia. }}{\longleftarrow} h_{5}^{A e o} \\
\text { beat }\left(a_{n}\right)=\text { off }
\end{array}\right. \\
& \left\{\begin{array}{l}
a_{n}=h_{3}^{\text {Aeo }} \\
a_{n+1}=h_{2}^{\text {Aeo }}, h_{4}^{\text {Aeo }} \\
a_{n+2}=s_{5}^{\text {Aeo }} 7 \\
a_{n+1}, a_{n+2} \in \text { bar } \\
\text { beat }\left(a_{n}\right)=\text { off }
\end{array} \Longrightarrow a_{n} \stackrel{\text { dia. }}{\longleftarrow} h_{5}^{\text {Aeo }}\right. \\
& \left\{\begin{array}{l}
a_{n}=h_{3}^{\text {Aeo }} \\
a_{n+1} \neq h_{2}^{\text {Aeo }}, h_{4}^{\text {Aeo }} \\
a_{n-1}=h_{2}^{\text {Aeo }}, h_{4}^{\text {Aeo }}, h_{7}^{\text {Aeo }} \\
a_{n-1}, a_{n} \in \text { bar }_{k}
\end{array} \Longrightarrow a_{n} \stackrel{\text { dia. }}{\longleftarrow} h_{5}^{\text {Aeo }}\right. \\
& \text { otherwise: } a_{n}=h_{3}^{\text {Aeo }} \stackrel{\text { dia. }}{\longleftarrow} h_{1}^{\text {Aeo }}
\end{aligned}
$$

19.3. (Inverse) DIATONIC Substitutions involving $h_{7}$

$$
\begin{aligned}
& \text { MAJOR Key }
\end{aligned}
$$

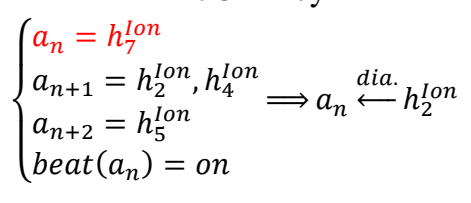

$$
\begin{aligned}
& \left\{\begin{array}{l}
a_{n}=h_{7}^{\text {Ion }} \\
a_{n+1}=h_{2}^{\text {Ion }}, h_{4}^{\text {Ion }} \\
a_{n+2}=h_{2}^{\text {Ion }}, h_{4}^{\text {Ion }} \Longrightarrow a_{n} \stackrel{\text { dia. }}{\longleftarrow} h_{2}^{\text {Ion }} \\
a_{n+3}=h_{5}^{\text {Ion }} \\
\text { beat }\left(a_{n}\right)=\text { on }
\end{array}\right. \\
& \text { otherwise: } a_{n}=h_{7}^{\text {Ion }} \stackrel{\text { dia. }}{\longleftarrow} h_{5}^{\text {Ion }}
\end{aligned}
$$

19.4. (Inverse) DIATONIC Substitutions involving $h_{2}$ and $h_{4}$

$$
\begin{gathered}
\text { MAJOR Key } \\
\left\{\begin{array}{l}
a_{n}=h_{2}^{\text {Ion }} \\
a_{n+1}=h_{1}^{\text {Ion }}, h_{4}^{\text {Ion }} \Longrightarrow a_{n} \stackrel{\text { dia. }}{\longleftarrow} h_{4}^{\text {Ion }}
\end{array}\right. \\
\left\{\begin{array}{l}
a_{n}=h_{4}^{\text {Ion }} \\
a_{n+1}=h_{2}^{\text {Ion }}, h_{5}^{\text {Ion }} \Longrightarrow a_{n} \stackrel{\text { dia. }}{\longleftarrow} h_{2}^{\text {Ion }}
\end{array}\right.
\end{gathered}
$$

\section{MINOR Key}

$$
\left\{\begin{array}{l}
a_{n}=h_{2}^{\text {Aeo }} \\
a_{n+1}=h_{1}^{\text {Aeo }}, h_{4}^{\text {Aeo }}
\end{array} \Longrightarrow a_{n} \stackrel{\text { dia. }}{\longleftarrow} h_{4}^{\text {Aeo }}\right.
$$$$
\left\{\begin{array}{l}
a_{n}=h_{4}^{A e o} \\
a_{n+1}=h_{2}^{A e o}, h_{5}^{A e o}, s_{5}^{A e o} 7
\end{array} \Longrightarrow a_{n} \stackrel{\text { dia. }}{\longleftarrow} h_{2}^{A e o}\right.
$$ 
Note - In this LOOP it is necessary to explicit the chord placed on the third beat.

In detail, a chord covering the beats from the first to the third must be rewritten and treated as two identical chords: the first chord covers the first two beats, the second exclusively covers the third. A chord covering the second and third beats must be rewritten and treated as two identical chords: the first chord covers the second beat, the second covers the third. A chord covering the beats from the second to the fourth must be rewritten and treated as two identical chords: the first chord exclusively covers the second beat, the second covers the last two.

\section{Further Inverse SECONDARY DOMINANTS Substitutions}

\section{MAJOR Key}

All the feasible Secondary Dominants Inverse Substitutions have already been carried out during phase 17.

$$
\begin{aligned}
& a_{n}=Y 7 \\
& a_{n}=s_{5}^{\text {Aeo }} 7 \Longrightarrow a_{n} \stackrel{\text { sec. dom. }}{\longleftarrow} h_{5}^{\text {Aeo }}
\end{aligned}
$$

EXTRA. Inverse TONICIZATION (to be applied every time it is possible)

Tonicization - Any (major seventh or minor seventh) chord, especially if it covers a certain number of consecutive bars, can be tonicized. The Tonicization, or Micro-Modulation, can be obtained by replacing a portion of the initial chord with a dominant seventh chord distant an ascending perfect fifth, so as to locally create a harmonically Perfect Cadence [1] [2] [3] [4] [7] [8].

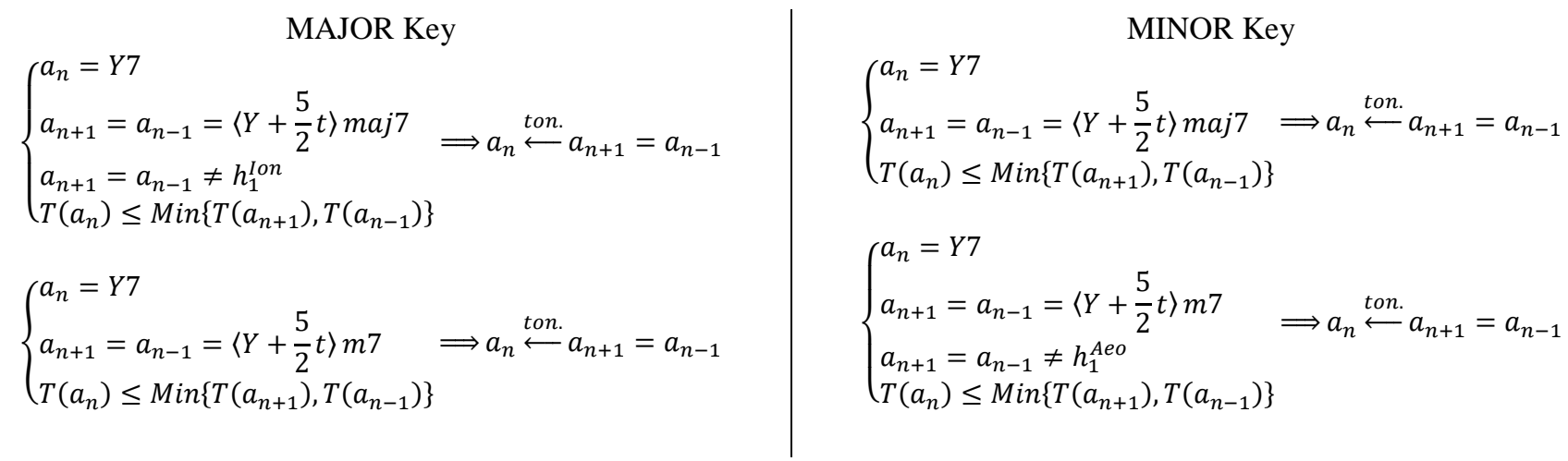

\section{Remarks}

It's worth underlining how chord progression analysis has almost nothing to do with the improvisation built on the original harmonic structure of a song. More precisely, net of a certain "Horizontalization" the musician can exploit by facing specific harmonic aggregates, such as "Tonicizations" and "Turnarounds", the improvisation should be carried out "vertically", abiding to the local harmony of the song.

The Local Tonal Centre can be characterized by significant fluctuations: sometimes, it is very difficult to identify it, in particular when a progression cannot be regarded as being manifestly "tonal" (built on cadences).

And especially in this case, paradoxically, the musician should improvise by abiding to the harmonic progression, chord by chord $[13-30]$.

\section{Acknowledgements}

I would like to thank my friend Luigi Serra, excellent guitarist and researcher at the University of Salerno, for having supported me in checking the validity of my transformations, and for having agreed to develop CAT CaSe together with me. CAT CaSe is an innovative android app, entirely based on the improved version of $C A T$ herein introduced, which allows to instantly carry out an accurate analysis of any chord progression. [https://play.google.com/store/apps/details?id=serra.cataldo.catcase] 


\section{References}

[1] Cataldo, C. (2018). Towards a Music Algebra: Fundamental Harmonic Substitutions in Jazz. International Journal of Advanced Engineering Research and Science, 5(1), 52-57. https://dx.doi.org/10.22161/ijaers.5.1.9

[2] Cataldo, C. (2018). Jazz e Sostituzioni Armoniche: Verso un Nuovo Formalismo - Jazz and Harmonic Substitutions: Towards a New Formalism. Journal of Science, Humanities and Arts (JOSHA), 5(1). https://dx.doi.org/10.17160/josha.5.1.381

[3] Cataldo, C. (2018). Extreme Chord Substitutions: a Qualitative Introduction to CAT (Cataldo Advanced Transformations). Journal of Science, Humanities and Arts (JOSHA), 5(4). https://dx.doi.org/10.17160/josha.5.4.424

[4] Cataldo, C. (2018). Music Algebra: Harmonic Progressions Analysis and CAT (Cataldo Advanced Transformations). International Journal of Advanced Engineering Research and Science, 5(5), 224-227. https://dx.doi.org/10.22161/ijaers.5.5.29

[5] Cork, C. (1988). Harmony by LEGO Bricks: A New Approach to the Use of Harmony in Jazz Improvisation. Leicester, United Kingdom: Tadley Ewing Publications.

[6] Cork, C. (2008). The New Guide to Harmony with LEGO Bricks. London: Tadley Ewing Publications.

[7] Levine, M. (2009). The Jazz Theory Book (Italian Edition by F. Jegher). Milan, IT: Curci Jazz.

[8] Cho, G. J. (1992). Theories and Practice of Harmonic Analysis. Lewiston, NY: E. Mellen Press.

[9] Schuijer, M. (2008). Analyzing Atonal Music: Pitch- Class Set Theory and Its Contexts. University of Rochester.

[10] Lewin, D. (1960). The Intervallic Content of a Collection of Notes, Intervallic Relations between a Collection of Notes and its Complement: an Application to Schoenberg's Hexachordal Pieces. Journal of Music Theory, 4(1), 98-101.

[11] Cataldo, C. (2018). A Simplified Introduction to Music Algebra: from the Scale Vectors to the Modal Tensor. International Journal of Advanced Engineering Research and Science, 5(1), 111-113. https://dx.doi.org/10.22161/ijaers.5.1.16

[12] Cataldo, C. (2018). Algebra Musicale: dai Vettori Scala al Tensore Modale - Music Algebra: from the Scale Vectors to the Modal Tensor. Journal of Science, Humanities and Arts (JOSHA), 5(1). https://dx.doi.org/10.17160/josha.5.1.383

[13] Cataldo, C. (2018). Breve Introduzione all'Improvvisazione Be-Bop [Short Introduction to Be-Bop Improvising]. Journal of Science, Humanities and Arts (JOSHA), 5(6). https://dx.doi.org/10.17160/josha.5.6.448

[14] Cataldo, C., Deidda, S., D’Errico, F., Martino, G. (2018). Definition and Usage of the Be-Bop Minor Scales (part 3): the BeBop Natural Minor Scale. Journal of Science, Humanities and Arts (JOSHA), 5(3). https://dx.doi.org/10.17160/josha.5.3.407

[15] Cataldo, C., Deidda, S., D’Errico, F., Martino, G. (2018). Definition and Usage of the Be-Bop Minor Scales (part 2): the BeBop Dorian Scale. Journal of Science, Humanities and Arts (JOSHA), 5(2). https://dx.doi.org/10.17160/josha.5.2.396

[16] Cataldo, C., Deidda, S., D’Errico, F., Martino, G. (2018). Definition and Usage of the Be-Bop Minor Scales (part 1): the BeBop Melodic Minor Scale. Journal of Science, Humanities and Arts (JOSHA), 5(2). https://dx.doi.org/10.17160/josha.5.2.395

[17] Cataldo, C., Martino, G. (2018). La Scala Maggiore Be-Bop: Definizione ed Utilizzo - The Be-Bop Major Scale: Definition and Usage. Journal of Science, Humanities and Arts (JOSHA), 5(2). https://dx.doi.org/10.17160/josha.5.2.393

[18] Cataldo, C., Martino, G. (2018). La Scala Dominante Be-Bop: Definizione ed Utilizzo - The Be-Bop Dominant Scale: Definition and Usage. Journal of Science, Humanities and Arts (JOSHA), 5(2). https://dx.doi.org/10.17160/josha.5.2.389

[19] Cataldo, C. (2017). Il Linguaggio Be-Bop e gli Accordi di Settima di Quarta Specie [The Be-Bop Language and The Major Seventh Chords]. Journal of Science, Humanities and Arts (JOSHA), 4(4). https://dx.doi.org/10.17160/josha.4.4.341

[20] Cataldo, C. (2017). The Art of Improvising: the Be-Bop Language and the Major Seventh Chords. Art and Design Review, 5, 222-229. https://doi.org/10.4236/adr.2017.54018

[21] Cataldo, C. (2017). Il Linguaggio Be-Bop e gli Accordi di Settima di Seconda Specie [The Be-Bop Language and The Minor Seventh Chords]. Journal of Science, Humanities and Arts (JOSHA), 4(4). https://dx.doi.org/10.17160/josha.4.4.339

[22] Cataldo, C. (2017). The Art of Improvising: the Be-Bop Language and the Minor Seventh Chords. Art and Design Review, 5, 213-221. https://doi.org/10.4236/adr.2017.54017

[23] Cataldo, C. (2017). Il Linguaggio Be-Bop e gli Accordi di Settima di Prima Specie [The Be-Bop Language and The Dominant Seventh Chords]. Journal of Science, Humanities and Arts (JOSHA), 4(4). https://dx.doi.org/10.17160/josha.4.4.340

[24] Cataldo, C. (2017). The Art of Improvising: the Be-Bop Language and the Dominant Seventh Chords. Art and Design Review, 5, 181-188. http://doi.org/10.4236/adr.2017.53014

[25] Wise. L. (1983). Bebop Bible - The Musicians Dictionary of Melodic Lines. United States: REH Publications.

[26] Coker, J., Casale, J., \& Campbell, G. (1982). Patterns for Jazz. Los Angeles, CA: Alfred Publishing Co. Inc.

[27] Baker, D. (1988). How to Play Bebop (Volume 1). Los Angeles, CA: Alfred Publishing Co. Inc.

[28] Baker, D. (1988). How to Play Bebop (Volume 2). Los Angeles, CA: Alfred Publishing Co. Inc.

[29] Baker, D. (1988). How to Play Bebop (Volume 3). Los Angeles, CA: Alfred Publishing Co. Inc.

[30] Baker, D. (1988). Jazz Improvisation. Los Angeles, CA: Alfred Publishing Co. Inc. 